\title{
Untersuchungen über die Veresterung unsymmetrischer zwei- und mehrbasischer Säuren.
}

II. Abhandlung:

Über die Veresterung der Brom- und der 0xyterephtalsäure,

von

Rud. Wegscheider und Karl Bittner.

Aus dem I. chemischen Laboratorium der k. k. Universität in Wien.

(Vorgelegt in der Sitzung am 15. Juni 1900.)

\section{A. Experimenteller Theil. ${ }^{1}$}

Im Anschlusse an die Versuche Wegscheiders über die Veresterung unsymmetrischer Dicarbonsäuren (Monatshefte, Bd. 16, 75; Bd. 18, 418 und 629; Bd. 20, 685) habe ich die Veresterung der Oxy- und der Bromterephtalsäure untersucht und theile im folgenden die Resultate mit.

I. Bromterephtalsäure.

Von Estern der Bromterephtalsäure war bisher nur der neutrale Methylester bekannt, der von Fischli (Ber., 12, 619) durch Einwirkung des Chlorids auf Holzgeist erhalten wurde.

Darstellung und Eigenschaften der Bromterephtalsäure.

Fischli (Ber., 12,619) stellte dieselbe durch Oxydation von 2-Brom-p-Toluylsäure mit Chamäleon dar. Eine andere Darstellungsmethode rührt von Fileti her (Gaz. chim., 16, 297), der die Säure beim Kochen von Bromcymol mit Salpetersäure erhielt. Ich bediente mich zur Herstellung der Bromterephtalsäure der Sandmeyer'schen Reaction, die es gestattet, eine

1 Von Karl Bittner. 
Amidogruppe gegen Brom auszutauschen. Als Ausgangsmaterial benützte ich die Amidoterephtalsäure, die ich aus der Nitrosäure nach dem Vorgange von Burkhardt (Ber., 10, 145) durch Reduction erhielt.

$5 g$ Nitrosäure wurden mit $8 g$ Zinn und $17 g$ concentrierter Chlorwasserstoffsäure reduciert. Die Reaction ist so lebhaft, dass beträchtliche Erwärmung eintritt, welche Kühlung nöthig macht. Wenn die freiwillige Erwärmung aufgehört hat, wird noch einige Zeit am Wasserbade erwärmt. Hiebei scheidet sich das Zinndoppelsalz als weiße compacte Masse aus, welche aber beim Verdünnen mit Wasser sofort unter Ausscheidung der gelben Amidosäure zerfällt. Zur Reinigung wird dieselbe mit Wasser gewaschen, dann in Salzsäure gelöst und mit essigsaurem Natrium wieder gefällt.

Diazotierung. Zuerst machte ich den Versuch so, dass

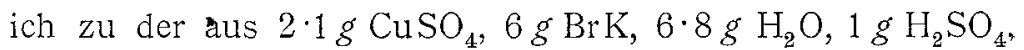
$3.5 \mathrm{~g}$ Kupferspänen bereiteten, bis zur Entfärbung gekochten Lösung von Kupferbromür $3 g$ Amidosäure zugab und dann $1.4 \mathrm{~g} \mathrm{NaNO} \mathrm{Na}_{2}\left(10_{0}^{\circ}\right.$ Überschuss), gelöst in Wasser, zutropfen ließ. Das erhaltene Product zeigte aber bloß einen Bromgehalt von $26.2 \%$, also ein Manco von $6.5 \%$. Es war dies ein Gemisch von Amido- und Bromterephtalsäure, dessen Trennung nicht gelang, da ihre Löslichkeitsverhältnisse nur sehr wenig verschieden sind. Folgende Methode erwies sich als die beste. $3 g$ der Amidosäure wurden, um sie in fein vertheiltem Zustande zu erhalten, in möglichst wenig gewöhnlicher Natronlauge gelöst und wieder mit Schwefelsäure gefällt. Dann wurde dieses Gemisch mit $10 \mathrm{~cm}^{3}$ einer $49 \%$ Bromwasserstoffsäure versetzt. und unter Eiskühlung langsam mit der obigen Menge Natriumnitrit versetzt, wobei die Temperatur $5^{\circ}$ nicht überschritt. Diese die diazotierte Amidosäure enthaltende Flüssigkeit wurde in die nach obiger Angabe bereitete Kupferbromürlösung eingetragen, wobei eine außerordentlich lebhafte Gasentwickelung zu bemerken ist. Wenn dieselbe nachlässt, so wird noch einige Zeit zur Erreichung einer vollständigen Umsetzung erwärmt. Die Flüssigkeit wurde dann verdünnt und ausgeäthert, wobei die Hauptmenge sofort in den Äther übergeht. Derselbe zeigte noch immer eine leichte Fluorescenz, was auf eine Verunreinigung 
durch Amidosäure schließen ließ. Der nach Abdunsten des Athers erhaltene Rückstand wurde dann behufs Reinigung einer fractionierten Fällung unterworfen. Das Rohproduct wurde in Kali gelöst, wobei die Lösung eine. dunkelrothe Färbung annimmt. Aus dieser Lösung habe ich drei bis vier Fractionen durch Fällung mit sehr verdünnter Salzsäure abgeschieden. Die erste war dunkel, die folgenden immer heller, bis endlich auf Zusatz von viel Salzsäure die Hauptmenge der Säure als weiße voluminöse Masse sich ausscheidet. Dieselbe wurde durch Umkrystallisieren aus heißem verdüntem Methylalkohol gereinigt und zeigte keine Fluorescenz mehr. Den Schmelzpunkt fand ich zu 301 bis $303^{\circ}$. Das Thermometer war durch Bestimmung des Siedepunktes von Diphenylamin $\left(301^{\circ}\right)$ geprüft worden. Er stimmt also fast genau mit dem, den Fischli angibt $\left(303\right.$ bis $304^{\circ}$ ). Die Analyse der bei $100^{\circ}$ getrockneten Substanz ergab Folgendes:

$0 \cdot 3307 g$ Substanz ergab $0.2527 g \mathrm{Br}$ Ag.

In 100 'Theilen:

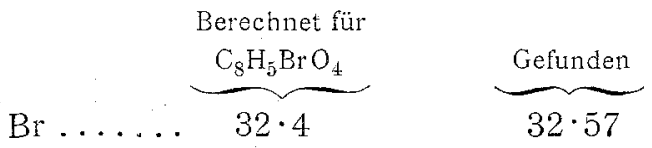

Aus den dunkleren Fractionen kann durch Vereinigung und Wiederholung der fractionierten Fällung noch eine kleine Menge allerdings nicht ganz weißer Säure erhalten werden.

Das saure Kalisalz lässt sich nicht auf dem gewöhnlichen Wege herstellen. Löst man Säure in der zur Herstellung des neutralen Salzes berechneten Menge Kali und trägt dann die gleiche Menge Säure ein, so erhält man nach eingetretener Lösung beim Einengen eine Krystallisation, die einen viel zu geringen Gehalt an Kalium ergibt $(0 \cdot 4 \%$ statt $13 \%)$.

\section{Esterification der Bromterephtalsäure.}

I. Mit Salzsäure.

a) $5 \mathrm{~g}$ der Säure wurden in $50 \mathrm{~cm}^{3}$ Methylalkohol gelöst, in diese Lösung trockenes Salzsäuregas bis zur Sättigung eingeleitet. Beim Erkalten scheidet sich ein Haufwerk von Krystallen aus; die Hauptmenge wird erst beim Verdünnen mit 
Wasser erhalten. Zur Reinigung wurde aus heißem verdünntem Methylalkohol umkrystallisiert. Ausbeute $4.5 \mathrm{~g}$ reiner Neutralester, Schmelzpunkt $54^{\circ}$.

b) $5 \mathrm{~g}$ Säure wurden in $50 \mathrm{~cm}^{3}$ Methylalkohol gelöst und mit $5 \mathrm{~cm}^{3}$ eines mit Salzsäuregas gesättigten Methylalkohols, der $0.4 \mathrm{~g} \mathrm{HCl} \mathrm{im} \mathrm{Cubikcentimeter} \mathrm{enthielt,} \mathrm{versetzt.} \mathrm{Nach} 24$ Stunden wurde die Lösung mit Wasser gefällt und die Fällung mit Benzol ausgekocht, um die gebildete Estersäure und Neutralester in Lösung zu bringen. Ungelöst blieb $2 \cdot 8 g$ unveränderte Säure. Das Benzol wurde abgedunstet, der Rückstand mit verdünntem Ammoniak verrieben, um die Estersäure in Lösung zu bringen. Ungelöst blieb $0.9 \mathrm{~g}$ Neutralester. Die ammoniakalische Lösung wurde mit Salzsäure gefällt. Die Fällung wurde aus heißem Wasser bis zum constanten Schmelzpunkte umkrystallisiert. Schmelzpunkt $164^{\circ}$. Ich erhielt $0.1 g$ an reiner Estersäure, welche ich $\beta$-Bromterephtalmethylestersäure nennen will.

$$
\text { II. Mit } \mathrm{H}_{2} \mathrm{SO}_{4} \text {. }
$$

a) $5 \mathrm{~g}$ Säure wurden in $50 \mathrm{~cm}^{3}$ Methylalkohol gelöst und mit $5 \mathrm{~cm}^{3} \mathrm{H}_{2} \mathrm{SO}_{4} \quad 1 \frac{1}{2}$ Stunden am Rückflusskühler erhitzt. Die Lösung wurde mit Wasser gefällt, die Fällung mit verdünntem Ammoniak behandelt. Ungelöst blieb $4 \cdot 3 g$ Neutralester. Die aus der Lösung mit Salzsäure erhaltene Fällung zeigte nach dem Umkrystallisieren den Schmelzpunkt $164^{\circ}$. Ein Gemisch dieser mit der beim vorigen Versuche erhaltenen schmilzt auch bei dieser Temperatur. Ausbeute $0.4 \mathrm{~g}$. Es entsteht also bei diesem Versuche ebenfalls $\beta$-Estersäure, wie bei dem Versuche mit Salzsäure.

b) $5 \mathrm{~g}$ Säure wurden in $30 \mathrm{~cm}^{3}$. Alkohol gelöst und mit dem gleichen Volum Schwefelsäure unter Vermeidung einer Erwärmung versetzt. Nach 24 Stunden war noch keine Reaction wahrzunehmen. Nach 4 Tagen war alles in Neutralester verwandelt worden.

\section{Einwirkung von Jodmethyl auf saures bromterephtalsaures Kali.}

Da ich das Kalisalz in fester Form nicht erhalten konnte, so nahm ich die Einwirkung des Jodmethyls, auf in Methyl- 
alkohol gelöstes Salz vor. $5 g$ Säure wurden in $50 \mathrm{~cm}^{3}$. Methylalkohol gelöst und mit der zur Bildung des sauren Kalisalzes nöthigen Menge wässerigen Kalis versetzt. ${ }^{1}$ Dazu wurde $5 g$ Jodmethyl gegeben und am Rückflusskühler durch 20 Stunden erhitzt. Die Lösung wurde dann mit Wasser gefällt, die Fällung mit verdünntem Ammoniak behandelt, worin sich alles löste, was auf Abwesenheit von Neutralester schließen ließ. Die Lösung wurde also wieder mit Salzsäure gefällt, die Fällung mit heißem Benzol behandelt, worin $4 g$ Säure ungelöst blieb. Die Lösung wurde abgedunstet. Der Rückstand, aus heißem Wasser umkrystallisiert, liefert $0.8 g$ einer Estersäure vom Schmelzpunkte $145^{\circ}$, die von der bei den vorigen Versuchen erhaltenen verschieden ist und die ich daher $\alpha$-Bromterephtalmethylestersäure nennen will.

\section{Einwirkung von Methylalkohol auf Bromterephtalsäure im Einschmelzrohre.}

Bei $100^{\circ}$ trat keine Einwirkung ein; ich nahm daher einen zweiten Versuch bei $150^{\circ}$ vor. $8 g$ Säure wurden $z u \quad 4 g$ in $30 \mathrm{~cm}^{3}$ Methylalkohol gelöst und im zugeschmolzenen Rohre durch 8 Stunden erhitzt. Aus der erhaltenen Lösung wurde dann der Alkohol abgedunstet und der Rückstand mit Benzol ausgekocht. Die aus der benzolischen Lösung erhaltene Krystallisation zeigt nach dem Umkrystallisieren den Schmelzpunkt $164^{\circ}$, war also $\beta$-Estersäure. Die Lauge wurde dann eingeengt und ergab noch $0: 6 \mathrm{~g}$ unreiner Estersäure. Die Endlauge wurde schließlich mit Ligroin gefällt und die Fällung mit verdünntem Ammoniak behandelt, worin Neutralester ungelöst blieb. Die aus der Lösung mit Salzsäure erhaltene Fällung zeigte den Schmelzpunkt 120 bis $125^{\circ}$. Da ich vermuthete, dass diese ein. Gemisch beider Estersäuren sei, versuchte ich, fractioniert zu fällen. Ich erhielt auch eine höher schmelzende Fraction, 143 bis $147^{\circ}$. Ein Gemisch dieser mit der Estersäure vom Schmelzpunkte $145^{\circ}$ schmolz aber schon bei 135 bis $137^{\circ}$, also zu tief, so dass sich nicht mit Sicherheit die Bildung auch der zweiten Estersäure bei diesem Versuche constatieren lässt.

1. Bei Anwendung von methylalkoholischem Kali scheidet sich Salz aus. 
Ausbeute $2 \cdot 3 g$ reine Estersäure, $0.6 g$ unreine, $2 g$ Neutralester, $1.4 \mathrm{~g}$ unveränderte Säure, Rest diese Gemische.

\section{Halbverseifung des Neutralesters mit Kalilauge.}

$5 \mathrm{~g}$ Neutralester wurden in $50 \mathrm{~cm}^{3}$ Methylalkohol gelöst und mit der zur Halbverseifung nöthigen Menge Kali am Rückflusskühler bis zum Eintritte der neutralen Reaction gekocht. Die Lösung wurde dann mit Wasser verdünnt und mit Äther zur Entfernung neutraler Substanzen, ausgeschüttelt. $0 \cdot 2 g$ Neutralester waren in diesen übergegangen. Das in der wässerigen Lösung vorhandene Kalisalz wurde dann mit Salzsäure zersetzt, wobei sich die freie Estersäure ausscheidet. Dieselbe wurde aus Wasser umkrystallisiert und hatte den Schmelzpunkt $145^{\circ}$, ist also a-Estersäure. Ausbeute $4 \cdot 4 g$ reiner Estersäure.

\section{Neutralester.}

Derselbe entsteht bei allen Esterificationsmethoden (ausgenommen die Einwirkung von Jodmethyl auf das saure Kalisalz) in größerer oder kleinerer Menge. Zur Darstellung desselben empfiehlt sich am besten die Esterification mit Salzsäure oder Erhitzen mit $\mathrm{H}_{2} \mathrm{SO}_{4}$. Derselbe zeigte im reinen Zustande immer den Schmelzpunkt $54^{\circ}$; Fischli fand ihn bei $42^{\circ}$, Fileti bei 52 bis $53^{\circ}$.

\section{Eigenschaften und Constitution der Estersäuren.}

Zum Unterschiede von der freien Säure zeigen die beiden Estersäuren ein bedeutend besseres Krystallisationsvermögen. Während man die erstere meistens anscheinend amorph, seltener deutlich krystallinisch erhält, gelingt es, die beiden Estersäuren beim langsamen Erkalten der heißen wässerigen Lösung in seidenglänzenden langen Nadeln zu erhalten. Sie sind beide in Alkohol leicht löslich, daraus durch Wasser fällbar, leicht 1öslich in Äther, heißem Wasser, Benzol, daraus durch Ligroin fällbar. Keine von ihnen zeigte einen Krystallwassergehalt. Die $\alpha$-Säure ist in kaltem Wasser schwerer löslich als die $\beta$-Säure. Die $\alpha$-Säure ist schneewei $\beta$, die $\beta$-Säure habe ich immer hellgelb erhalten. Ich lasse nun ihre Analyse folgen. 
Die bei $100^{\circ}$ getrockneten Substanzen ergaben:

$\alpha$-Säure. $0.2043 g$ Substanz ergab $0.3128 g \quad \mathrm{CO}_{2}$ und $0.0552 \mathrm{~g} \mathrm{H}_{2} \mathrm{O}$.

In 100 Theilen:
Berechnet für

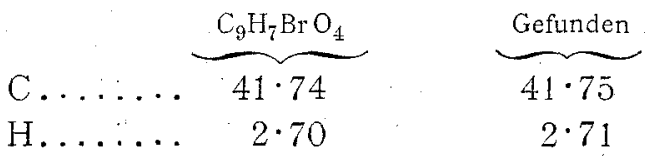

$\beta$-Säure. $0 \cdot 1947 \mathrm{~g}$ Substanz ergab bei der Methoxylbestimmung $0 \cdot 1812 \mathrm{~g} \mathrm{AgJ}$.

In 100 Theilen:

$\mathrm{OCH}_{3} \ldots \underbrace{\begin{array}{c}\text { Berechnet für } \\ \mathrm{C}_{9} \mathrm{H}_{7} \mathrm{BrO}_{4}\end{array}}_{12 \cdot 36} \quad \underbrace{\text { Gefunden }}_{12 \cdot 27}$

Ich habe mit der Bromterephtalsäure und den beiden Estersäuren Fällungen mit Metallsalzlösungen vorgenommen, und zwar so, dass ich einprocentige, mit $\mathrm{NH}_{3}$ neutralisierte Löstungen der Säuren herstellte. Das Resultat zeigt die folgende Tabelle.

\begin{tabular}{|c|c|c|c|c|c|}
\hline & Bleiacetat & $\mathrm{CuSO}$ & $\mathrm{AgNO}$ & $\mathrm{FeCi}_{3}$ & $\mathrm{Hg}\left(\mathrm{NO}_{3}\right)_{2}$ \\
\hline $\begin{array}{l}\text { Freie } \\
\text { Säure }\end{array}$ & $\begin{array}{l}\text { Niederschlag, } \\
\text { im Über- } \\
\text { schusse } \\
\text { löslich, fällt } \\
\text { beim Kochen } \\
\text { sofort wieder } \\
\text { aus }\end{array}$ & $\begin{array}{l}\text { Allmählich } \\
\text { Fällung }\end{array}$ & $\begin{array}{l}\text { Nieder- } \\
\text { schlag }\end{array}$ & $\begin{array}{l}\text { Bräunliche } \\
\text { Fällung }\end{array}$ & $\begin{array}{l}\text { Leichte } \\
\text { Trübung }\end{array}$ \\
\hline $\begin{array}{l}\beta \text {-Ester- } \\
\text { säure }\end{array}$ & Dasselbe & $\begin{array}{l}\text { Keine } \\
\text { Fällung }\end{array}$ & $\begin{array}{l}\text { Allmählich } \\
\text { Fällung }\end{array}$ & $\begin{array}{c}\text { Weiße } \\
\text { Fällung }\end{array}$ & $\begin{array}{l}\text { Reichliche } \\
\text { Fällung }\end{array}$ \\
\hline $\begin{array}{l}\alpha \text {-Ester- } \\
\text { säure }\end{array}$ & $\begin{array}{l}\text { Fällt beim } \\
\text { Kochen nicht } \\
\text { aus }\end{array}$ & $\begin{array}{l}\text { Allmählich } \\
\text { Fällung }\end{array}$ & $\begin{array}{c}\text { Reichliche } \\
\text { Fällung }\end{array}$ & $\begin{array}{l}\text { Weiße } \\
\text { Fällung }\end{array}$ & $\begin{array}{l}\text { Reichliche } \\
\text { Fällung }\end{array}$ \\
\hline
\end{tabular}

Mit $\mathrm{Hg} \mathrm{Cl}_{2}$ habe ich keine Fällungen erhalten. 
Für die Darstellung der a-Estersäure eignet sich die Halbverseifung des Neutralesters am besten, für die Darstellung der $\beta$-Estersäure Erhitzen der freien Säure mit Methylalkohol im Einschmelzrohr. Zur Ermittelung der Constitution der beiden Estersäuren habe ich noch ihre Silbersalze hergestellt. Dazu habe ich die Estersäuren in der berechneten Menge verdünnten Ammoniaks gelöst und mit Silbernitrat gefällt. Zur Reinigung habe ich die Salze aus heißem Wasser umkrystallisiert, aus dem sie sich beim Erkalten in weißen Flocken ausscheiden.

Zur Analyse wurden die Salze bei $100^{\circ}$ getrocknet. Das Silber wurde bestimmt, indem die getrockneten Salze zuerst an der Luft und dann im Rose'schen Tiegel im Wasserstoffstrome geglüht wurden.

Silbersalz der $\alpha$-Säure: $0.2685 g$ Substanz ergab $0.0797 g$ metallisches Silber.

In 100 Theilen:

$$
\underbrace{\begin{array}{l}
\text { Berechnet für } \\
\mathrm{C}_{9} \mathrm{H}_{6} \mathrm{AgBr} \mathrm{BrO}_{4}
\end{array}}_{29 \cdot 39} \quad \underbrace{\text { Gefunden }}_{29 \cdot 69}
$$

Silbersalz der $\beta$-Säure: $0.2437 \mathrm{~g}$ Substanz ergab $0.0718 \mathrm{~g}$ Silber.

In 100 Theilen:

$$
\frac{\text { Berechnet }}{29 \cdot 39} \quad \frac{\text { Gefunden }}{29 \cdot 46} \text {. }
$$

Die Zersetzung der Silbersalze wurde in einer Eprouvette im Luftstrome mit vorgelegter Kalilauge und folgendem Barytwasser vorgenommen; bei $220^{\circ}$ begann sich letzteres zu trüben, auch machte sich der charakteristische Geruch aromatischer Bromkörper geltend. Doch konnte im Rückstande der Eprouvette nach der Verseifung desselben nur Bromterephtalsäure constatiert werden. Selbst beim Erhitzen auf $300^{\circ}$ im Eisenfeilbade war kein anderes Resultat zu erzielen. Schließlich versuchte ich, durch Destillation der freien Estersäuren Kohlensäure abzuspalten, machte aber die interessante Wahrnehmung, 
dass dieselben unzersetzt destillieren. Die $\alpha$-Säure siedet unter $37 \mathrm{~mm}$ Druck bei $235^{\circ}$, die $\beta$-Säure unter $19 \mathrm{~mm}$ Druck bei $233^{\circ}$. Dass dieselben in der That unzersetzt destillieren, beweist der Umstand, dass sie nach dem Umkrystallisieren den richtigen Schmelzpunkt zeigten und nach der Verseifung Bromterephtalsäure zurückgeben.

Nachdem die Versuche, die Silbersalze zu zersetzen, keine Aufschlüsse über die Constitution der beiden Isomeren gaben, so ist für dieselbe bloß die elektrische Leitfähigkeitsconstante. entscheidend. Die Bestimmung derselben, die ich Herrn Prof. Wegscheider zu verdanken habe, ergab folgende Werte:

Für die $\alpha$-Säure $K=0.037$.

$$
\text { » } \beta \text {-Säure } K=0.50 \text {. }
$$

Die Constante der letzteren ist also mehr als zehnmal so groß als die der ersteren. Dies ist auch der Grund, warum ich entsprechend dem Vorschlage Wegscheiders (Monatshefte, 16,141 ), die bei $145^{\circ}$ schmelzende Estersäure als o-Säure bezeichnet habe. Da nach Analogie mit den Brombenzoesäuren jene Estersäure die stärkere sein muss, die das freie Carboxyl in Orthostellung zum Brom hat, so ergibt sich folgende Constitution für die beiden Estersäuren:
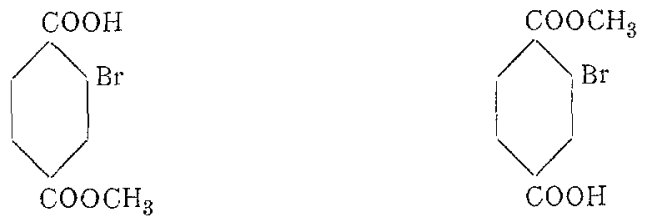

B-Bromterephtalmethylestersäure.

$\alpha$-Bromterephtalmethylestersäure.

\section{0xy terephtalsäure.}

Ich habe dieselbe nach der Vorschrift von Burkhardt (Ber., 10, 145) hergestellt. Derselbe machte sie aus Amidosäure durch Diazotieren und Kochen mit Wasser. Beim Diazotieren ist jedoch stets darauf $z u$ achten, dass die Schwefelsäure im Überschusse vorhanden ist, da sonst Dunkelfärbung der Substanz eintritt. Diese ist jedoch leicht durch Entfärben der alkalischen Lösung mit Thierkohle zu beseitigen. Zur vollständigen Reinigung habe ich auch hier die fractionierte Fällung 
vorgenommen, doch gelang es nicht, ein vollständig weißes Product zu erhalten; dasselbe zeigt einen gelblichen Stich.

\section{Esterification der Oxyterephtalsäure.}

\section{Mit $\mathrm{HCl}$.}

a) Durch Einleiten von Salzsäuregas durch 2 Stunden in die methylalkoholische Lösung der Säure entsteht, wie schon Burkhardt gezeigt hat, ausschließlich Neutralester.

b) $5 \mathrm{~g}$ Säure wurden in $100 \mathrm{~cm}^{2}$ Methylalkohol gelöst und mit $5 \mathrm{~cm}^{2}$ eines mit Salzsäuregas gesättigten Methylalkohols versetzt. Nach 24 Stunden wurde die Lösung mit Wasser gefällt. In der Fällung konnte aber weder Neutralester, noch Estersäure constatiert werden.

$$
\text { II. Mit } \mathrm{H}_{2} \mathrm{SO}_{4} \text {. }
$$

a) $4 \mathrm{~g}$ Säure wurden in $100 \mathrm{~cm}^{3}$ Methylalkohol gelöst und mit $5 \mathrm{~cm}^{3} \mathrm{H}_{2} \mathrm{SO}_{4} 1 \frac{1}{2}$ Stunden am Rückflusskühler erhitzt. Die Lösung wurde mit Wasser gefällt und die Fällung mit verdünntem Ammoniak behandelt. Ungelöst blieb $1.7 \mathrm{~g}$ Neutralester. Die Lösung wurde mit Salzsäure gefällt, die Fällung aus heißem verdünntem Methylalkohol umkrystallisiert. Schmelzpunkt $177^{\circ}$. Das Product ist eine Estersäure, die ich $\beta$-Oxyterephtalmethylestersäure nennen will.

b) $4 \mathrm{~g}$ Säure wurden in $80 \mathrm{~cm}^{3}$ Methylalkohol gelöst und mit dem gleichen Volum Schwefelsäure in der Kälte versetzt. Nach 24 Stunden schied sich ein Haufwerk von Krystallen aus, das sich als Neutralester entpuppte. Weitere Mengen konnten noch durch Verdünnen der Lauge mit Wasser erhalten werden. Bildung von Estersäure konnte bei diesem Versuche nicht beobachtet werden.

\section{Verseifung des Neutralesters mit Kali.}

$4 \mathrm{~g}$ Neutralester wurden in methylalkoholischer Lösung mit der zur Halbverseifung nöthigen Menge Kali versetzt und bis zur neutralen Reaction am Rückflusskühler erhitzt. Die Lösung wurde dann mit Wasser verdünnt, ausgeäthert, wobei 
$0.6 g$ Neutralester in den Äther giengen, schließlich mit Salzsäure gefällt. Die Fällung wurde dann mit Benzol ausgekocht, worin die freie Säure fast unlöslich ist. Nach Umkrystallisieren aus verdünntem Methylalkohol zeigte das in Benzol Lösliche dem Schmelzpunkt $177^{\circ}$. Dass diese Substanz mit der bei Versuch II $a$ erhaltenen Estersäure identisch ist, wurde dadurch bewiesen, dass ein Gemisch beider ebenfalls den Schmelzpunkt $177^{\circ}$ zeigt. Ausbeute $2.8 \mathrm{~g}$ reine Estersäure.

\section{Einwirkung von Jodmethyl auf saures oxyterephtalsaures Kali.}

Die Darstellung des sauren Kalisalzes gelingt bei der Oxyterephtalsäure nach der üblichen Methode. $5 \mathrm{~g}$ Säure habe ich in der zur Bildung des neutralen Salzes berechneten Menge Kalilauge gelöst, hierauf weitere $5 g$ Säure hinzugegeben. Beim Einengen tritt Ausscheidung ein. Von dieser habe ich, nachdem sie lufttrocken war, eine Krystallwasserbestimmung gemacht.

Bei $140^{\circ}$ verlor $0.2648 \mathrm{~g}$ Substanz $0.0190 \mathrm{~g}$.

In 100 Theilen:

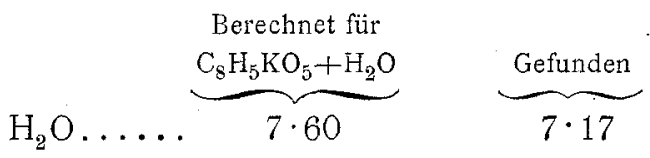

Dieser Gehalt entspricht einem Molecüle Krystallwasser. Analyse der bei $140^{\circ}$ getrockneten Substanz:

$0.2657 g$ Substanz ergab 0.1000 g $\mathrm{K}_{2} \mathrm{SO}_{4}$.

In 100 Theilen:

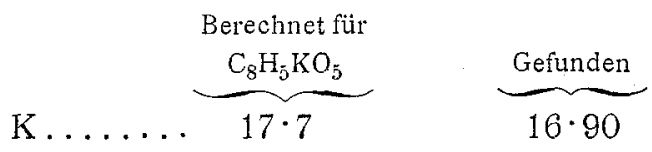

$10 \mathrm{~g}$ des Kalisalzes wurden mit Methylalkohol überschichtet und mit $10 \mathrm{~g}$ Jodmethyl am Rückflusskühler durch 20 Stunden erhitzt. Die alkoholische Lösung wurde dann von dem unzersetzten Kalisalze getrennt und abgedunstet. Der Rückstand wurde mit Benzol ausgekocht. Aus der benzolischen Lösung erhielt ich eine Krystallisation, die nach der Reinigung 
den Schmelzpunkt 206 bis $208^{\circ}$ zeigte. Ausbeute $0.2 \mathrm{~g}$. Es war eine von der früheren verschiedene Estersäure, die ich a-Cxyterephtalmethylestersäure nennen will. Ein Gemisch dieser mit der $\beta$-Säure schmolz bei 170 bis $172^{\circ}$.

\section{Einwirkung von Methylalkoho1 im Rohre.}

$5 \mathrm{~g}$ Säure wurden mit $30 \mathrm{~cm}^{3}$ Methylalkohol überschichtet und im Einschmelzrohre auf $180^{\circ}$ durch 8 Stunden erhitzt. Der Alkohol wurde abgedunstet und der Rückstand mit Benzol ausgekocht. Ungelöst blieb $2 \cdot 6 \mathrm{~g}$ Säure. Die benzolische Lösung wurde dann abgedunstet, der Rückstand mit verdünntem $\mathrm{NH}_{3}$ behandelt; ungelöst blieb $0.6 \mathrm{~g}$ Neutralester. Aus der Lösung erhielt ich durch Salzsäure eine Substanz, die nach der Reinigung den Schmelzpunkt $177^{\circ}$ zeigt, also mit der $\beta$-Säure identisch ist, was auch durch den Schmelzpunkt eines Gemisches $\left(177^{\circ}\right)$ bewiesen wird.

\section{Eigenschaften und Constitution der Estersäuren.}

Beide sind in heißem Wasser leichter löslich als die freie Säure, löslich in warmem Benzol, in Äther und Alkohol leicht löslich, aus Alkohol durch Wasser fällbar. Sie krystallisieren in feinen weißen Nadeln. Die $\beta$-Säure ist in $\mathrm{CHCl}_{3}$ leichter löslich als die andere. Analysen:

$\alpha$-Estersäure: $0.2277 g$ der bei $100^{\circ}$ getrockneten Substanz ergab $0.2638 \mathrm{~g} \mathrm{AgJ}$.

In 100 Theilen:

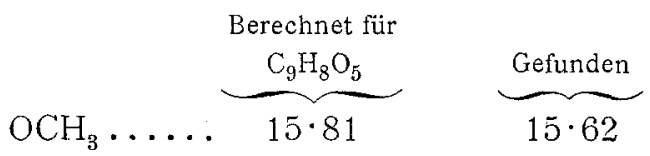

$\beta$-Estersäure: $0.1501 \mathrm{~g}$ der bei $100^{\circ}$ getrockneten Substanz ergab $0.1709 \mathrm{~g} \mathrm{AgJ}$.

In 100 Theilen:

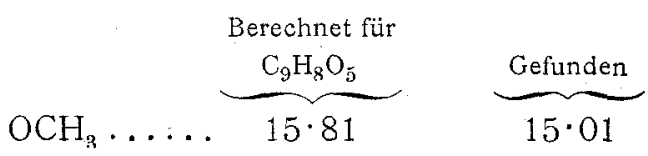


Die Silbersalze habe ich nach derselben Methode wie bei der Bromterephtalsäure hergestellt.

Analyse des bei $100^{\circ}$ getrockneten Salzes der $\beta$-Estersäure: $0 \cdot 2881 g$ ergab $0 \cdot 0811 g$ Silber.

In 100 Theilen:

$$
\mathrm{Ag} \ldots \ldots \ldots \underbrace{\begin{array}{c}
\text { Berechnet für } \\
\mathrm{C}_{9} \mathrm{H}_{7} \mathrm{Ag} \mathrm{O}_{5}
\end{array}}_{35 \cdot 88} \quad \underbrace{\text { Gefunden }}_{35 \cdot 55}
$$

Die Zersetzung des Silbersalzes habe ich unter denselben Bedingungen vorgenommen wie bei der Bromterephtalsäure. Es war der Geruch von Phenol wahrzunehmen; aber trotz der hohen Temperatur von $300^{\circ}$ blieb das Salz zum größten Theile unzersetzt.

Indessen ist die Constitution der beiden isomeren Estersäuren durch ihr verschiedenes Verhalten gegen Eisenchlorid bis zu einem hohen Grade von Wahrscheinlichkeit sichergestellt. Während nämlich die Estersäure vom Schmelzpunkte $177^{\circ}$ die purpurviolette Eisenreaction der freien Säure zeigt, gibt die andere eine gelbrothe Färbung. Verseift man aber die letztere, so zeigt sie natürlich die purpurviolette Eisenreaction der Oxyterephtalsäure. Nimmt man also an, dass das Auftreten der purpurrothen Färbung an das zur Carboxylgruppe benachbarte Hydroxyl gebunden ist, wie es bei der Salicylsäure im Gegensatze zur $m$-Oxybenzoesäure der Fall ist, so ergibt sich folgende Constitution:

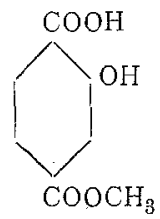

$\beta$-Estersäure.

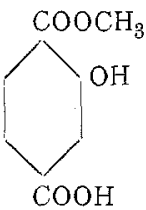

$\alpha$-Estersäure.

Endlich unterscheidet sich auch die Salicylsäure von den beiden anderen Oxybenzoesäuren durch ihre leichte Löslichkeit in Chloroform. Da nun auch die $\beta$-Säure in Chloroform viel leichter löslich ist als die $\alpha$-Säure, so ist die von mir angenommene Constitution sehr wahrscheinlich. Leider hatte ich 
von der $a$-Säure nur so wenig erhalten, dass für die Leitfähigkeitsbestimmung nichts übrig blieb. Da aber nach der Analogie mit den Oxybenzoesäuren nicht zu bezweifeln ist, dass jene Estersäure die größere Dissociationsconstante hat, in der das freie Carboxyl dem Hydroxyl benachbart ist, befindet sich die gewählte Benennung jedenfalls im Einklange mit den von Wegscheider vorgeschlagenen Bezeichnungen.

Anhang. Geleitet von dem Bestreben, die Constitution der Oxyterephtalestersäuren festzustellen, habe ich es versucht, vermittels der Schmitt'schen Reaction durch Einwirkung von Kohlensäure auf das Natriumsalz des $m$-Oxybenzoesäuremethylesters eine Oxyterephtalestersäure zu erhalten. Dazu habe ich den noch unbekannten Methylester der $m$-Oxybenzoesäure dargestellt. Ich erbielt ihn durch Erhitzen der methylalkoholischen Lösung der Säure mit Schwefelsäure. Verdünnt man die Lösung mit Wasser, so scheidet sich ein Öl ab. Ich habe die Flüssigkeit ausgeäthert; nach Abdunsten des Äthers hinterblieb ein Öl, welches nach längerem Stehen krystallinisch erstarrte. Zur Reinigung habe ich in wenig Benzol gelöst, worin sich der Äther leicht löste, und mit Ligroin versetzt. Nach einiger Zeit scheidet er sich in prachtvoll rechteckigen Blättchen aus. Schmelzpunkt $69^{\circ}$.

Zur Herstellung des Natriumsalzes habe ich ihn in absolutem Alkohol gelöst und diese Lösung mit der in absolutem Alkohol gelösten, berechneten Menge Natriumäthylat versetzt. Der Alkohol wurde im Vacuum abgedunstet; das Salz hinterbleibt als rein weiße Masse. Es wurde noch weiter bei $100^{\circ}$ im Vacuum getrocknet.

Der Versuch, Carboxyl einzuführen, misslang, da Verseifung des Natriumsalzes eintrat.

Da Haehle die Schmitt'sche Reaction mit dem Äthylester durchführen konnte, ${ }^{1}$ wird sie wohl auch beim Methylester durchführbar sein, wenn Wasserspuren vollständig ausgeschlossen sind. Feuchtes Natriumsalz verseift sich schon bei $100^{\circ}$. Da aber auch eine Wanderung der Methylgruppe bei höherer Temperatur nicht ausgeschlossen ist und die Synthese

1 Journal für prakt. Chemie, N. F., 44, 14. 
einer Oxyterephtalestersäure auf diesem Wege daher doch keinen völlig unanfechtbaren Beweis für die Constitution geliefert hätte, habe ich den Versuch nicht weiter verfolgt.

\section{B. Theoretischer Theil.}

Sowohl bei der Brom-, als bei der Oxyterephtalsäure ist das dem Brom, respective Hydroxyl benachbarte Carboxyl das stärkere und zugleich dasjenige, bei dem die sterischen Hinderungen stärker wirksam sind. Es ist daher bei den substituierten Terephtalsäuren ein ähnliches Verhalten hinsichtlich der Veresterung zu erwarten wie bei der Hemipinsäure.

Nach den von mir (Monatshefte, 16, 141; 18,640) aufgestellten Regeln hängt die Esterbildung aus sauren Salzen und Jodalkyl von der Stärke der Carboxyle ab, und zwar wird das stärkere verestert. Es entstehen also $\alpha$-Estersäuren. Diese Regel war bisher nur durch das Verhalten der Hemipinsäure (Wegscheider, Monatshefte, 16,131) gestützt. Bèi der Camphersäure, deren Carboxyle sich hinsichtlich der-Dissociierbarkeit nicht sehr unterscheiden, waren beide Estersäuren erhalten worden (Wegscheider, Monatshefte, 20, 685). Die von Bittner untersuchten Säuren gaben ebenfalls $\alpha$-Estersäuren, wodurch die Regel bestätigt wird.

Bei der Veresterung mit Salzsäure und Alkohol soll nach einer von mir aufgestellten Regel jenes Carboxyl zuerst verestert werden, bei dem die sterischen Hinderungen weniger wirksam sind. Bei den substituierten Terephtalsäuren ist daher wie bei der Hemipinsäure und im Gegensatze zur Camphersäure die Esterification des schwächeren Carboxyls, also Bildung von $\beta$-Estersäuren zu erwarten. Das wurde in der That bei der Bromterephtalsäure beobachtet; bei der Oxyterephtalsäure wurde auf diesem Wege nur Neutralester erhalten, doch werden sich wohl auch Bedingungen ermitteln lassen, unter denen auch Estersäure entsteht.

Für die Veresterung mit Schwefelsäure und.Alkohol habe ich dieselbe Regel gegeben wie für Salzsäure. Das hat sich

1 Von Rud. Wegscheider. 
auch bei den untersuchten Säuren bestätigt, indem auf diesem Wege $\beta$-Estersäuren erhalten wurden.

Bei der Anwendung von viel Schwefelsäure wurde nur Neutralester erhalten. Die von mir (Monatshefte, 18, 652) aufgeworfene Frage, ob bei Anwendung von viel Schwefelsäure ein specifischer, von der katalytischen Wirkung der Wasserstoffionen verschiedener Einfluss der Schwefelsäure in Betracht kommt, hat daher durch die Versuche Bitttners keine Beantwortung erfahren.

Die Verseifung des Neutralesters mit Alkalien soll nach einer von mir (Monatshefte, 16, 147) aufgestellten Regel Estersäuren geben, welche mit den mit Alkohol und Salzsäure oder Schwefelsäure entstehenden isomer sind. Das ist in der That bei der Bromterephtalsäure der Fall, welche bei dieser Reaction $\alpha$-Estersäure gibt. Ich und bald darauf auch V. Meyer haben hervorgehoben, dass die Verseifung ebenso wie die Veresterung mit Salzsäure und Alkohol von den sterischen Hinderungen abhängt, so dass das schwerer zu esterificierende Carboxyl auch schwerer verseift wird. Daraus folgt nothwendig die von mir aufgestellte Regel. Indes habe ich seither aufmerizsam gemacht (Monatshefte, 18, 634), dass der Satz von der Analogie zwischen der Veresterung mit Salzsäure und der Verseifung mit Kali einer Einschränkung bedarf. Hiefür liefert die Oxyterephtalsäure einen experimentellen Beleg. Sie ist die erste unsymmetrische Dicarbonsäure, die bei der Veresterung mit Schwefelsäure und bei der Verseifung des Neutralesters dieselbe Estersäure liefert. Beim Oxyterephtalsäureester wird jenes Carboxyl zuerst verseift, bei dem die sterischen Hinderungen stärker wirksam sind. In welcher Weise der Satz von der Beeinflussung der Verseifung durch sterische Hinderungen einzuschränken ist, wird sich erst an der Hand eines größeren Beobachtungsmateriales ermitteln lassen.

Ich habe auch die Frage aufgeworfen (Monatshefte, 18, 655), ob die Esterbildung aus Säure und Alkohol bei Gegenwart starker Säuren (indirecte Esterbildung) in derselben Weise von der Constitution des Säuremolecüls abhängt, wie die Esterbildung aus Säure und Alkohol allein (directe Esterbildung). Bei der Hemipinsäure geben die directe und die 
indirecte Esterbildung verschiedene Estersäuren. Ich habe hervorgehoben, dass aus diesem Resultate nicht auf eine wesentliche Verschiedenbeit der directen und der indirecten Esterbildung geschlossen werden kann, sondern dass das Verhalten der Hemipinsäure wahrscheinlicher als ein durch intermediäre Anhydridbildung bedingter Ausnahmsfall aufzufassen ist. Diese Auffassung wird durch Bittners Versuche bestätigt. Bei beiden untersuchten substituierten Terephtalsäuren liefert die directe, wie die indirecte Methode $\beta$-Estersäuren. (Bei der Bromterephtalsäure ist allerdings die Bildung der Isomeren in kleiner Menge nicht ausgeschlossen.) Es wird also auch bei der directen Esterbildung jenes Carboxyl zuerst esterificiert, welches weniger durch sterische Hinderungen beeinflusst ist. Daher kann bei der directen Esterbildung entsprechend der Henry'schen Hypothese eine intermediäre Anlagerung von Alkohol an die Carbonylgruppe angenommen werden.

\section{Zusammenfassung.}

Es wurde eine neue Darstellungsmethode der Bromterephtalsäure aus Amidosäure beschrieben. Ferner wurde die Esterification der Brom- und der Oxyterephtalsäure mit Methylalkohol allein und bei Gegenwart von Salzsäure und Schwefelsäure, ferner die Einwirkung von Jodmethyl auf die sauren Kalisalze dieser Säuren, endlich die unvollständige Verseifung der Neutralester untersucht. Hiebei wurden die beiden isomeren Methylestersäuren beider Säuren zum erstenmale dargestellt. Ferner wurden Angaben über den $m$-Oxybenzoesäuremethylester gemacht. Die erhaltenen Resultate stimmen mit den Wegscheider'schen Regeln über die Veresterung unsymmetrischer Dicarbonsäuren überein. Nur bei der Verseifung des Neutralesters der Oxyterephtalsäure tritt eine (von Wegscheider vorausgesehene) Ausnahme ein. Für die Esterbildung aus Säure und Alkohol allein gelten dieselben Regeln wie für die bei Gegenwart starker Säuren. 